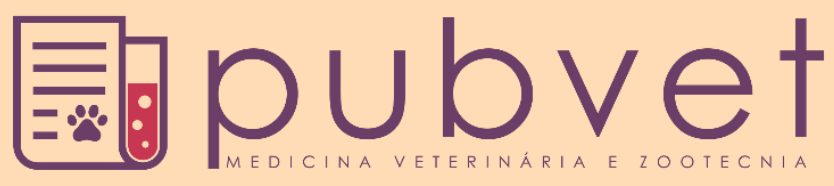

https://doi.org/10.31533/pubvet.v15n01a733.1-8

\title{
Hiperadrenocorticismo em felino: Relato de caso
}

\author{
Mariana Cosentino Mandaro ${ }^{1 *}$, Carolina Figueiredo Viegas ${ }^{1}$, Larissa Pagano Gama ${ }^{19}$, Milena \\ Fernandes Brandão de Oliveira ${ }^{1}$, Georgia Fernandes Barreto ${ }^{1}$, Flavia Tavares Manoel$^{2}$, Fabiana \\ Batalha Knackfuss ${ }^{30}$
}

${ }^{1}$ Acadêmica de Medicina Veterinária, Universidade do Grande Rio - UNIGRANRIO. Duque de Caxias - RJ, Brasil.

${ }^{2}$ Professora do Curso de Pós Graduação em Endocrinologia da Anclivepa São Paulo e fundadora da Associação Brasileira de Endocrinologia Veterinária (ABEV). Rio de Janeiro-RJ, Brasil.

${ }^{3}$ Professora Adjunta do Curso de Medicina Veterinária da Universidade do Grande Rio (UNIGRANRIO), Duque de Caxias - RJ, Brasil.

*Autor para correspondência, E-mail: mari_mandaro@hotmail.com

Resumo. O Hiperadrenocorticismo (HAC) é uma doença endócrina consequente da exposição excessiva ao cortisol, podendo ser decorrente da alteração funcional das glândulas adrenais ou da administração crônica de glicocorticoides. Essa patologia em felinos é rara e acomete, em maioria, diabéticos de meia idade a idade avançada. No presente relato o caso descrito é de um felino, macho, pelo curto brasileiro, com 16 anos, com pré-diagnóstico de Diabetes Mellittus e manifestando, dentre outros, os seguintes sintomas: prostração, vômito, palidez das mucosas, dilatação abdominal e pele delgada e sem elasticidade. O volume abdominal e a condição da pele associados a diabetes descompensada sugeriram a possibilidade de diagnóstico, com confirmação final mediante o Teste de Supressão com Dexametasona. Foi estabelecido terapia com o uso do Trilostano, que se mostrou eficaz no controle da patologia. O objetivo do relato de caso é mostrar que o hiperadrenocorticismo também acomete felinos, sendo uma das causas a diabetes na espécie.

Palavras-chave: Felino, hiperadrenocorticismo, trilostano

\section{Hyperadrenocorticism in feline: Case report}

Abstract. Hyperadrenocorticism (CAH) is an endocrine disease resulting from excessive exposure to cortisol and can be due to functional changes in the adrenal glands or chronic administration of glucocorticoids. This pathology in felines is rare and affects, in most of the cases, diabetics from middle age to old age. In the present report, the case described is of a feline, male, Brazilian Shorthaired Cat, aged 16, with pre-diagnosis of Diabetes Mellitus and manifesting, among others, the following symptoms: prostration, vomiting, mucous pallor, abdominal dilation, and thin and inelastic skin. The abdominal volume and skin condition associated with decompensated diabetes suggested the possibility of diagnosis, with final confirmation using the Dexamethasone Suppression Test. Therapy was established with the use of Trilostane, which proved to be effective in the control of the pathology. The objective of the case report is to show that hyperadrenocorticism also affects cats, being one of the causes of diabetes in the species.

Keywords: Felines, hyperadrenocorticism, trilostane

\section{Hiperadrenocorticismo en felino: Reporte de un caso}

Resumen. El hiperadrenocorticismo (HAC) es una enfermedad endocrina resultante de la exposición excesiva al cortisol y puede deberse a cambios funcionales en las glándulas suprarrenales o la administración crónica de glucocorticoides. Esta patología en felinos es 
rara y afecta, en su mayoría, diabéticos de mediana edad a edad avanzada. En el presente informe, el caso descrito es el de un felino, macho, gato brasileño de pelo corto, de 16 años, con prediagnóstico de Diabetes Melittus y manifestando, entre otros, los siguientes síntomas: postración, vómitos, palidez de mucosas, dilatación abdominal y piel delgada y sin elasticidad. El volumen abdominal y la condición de la piel asociada con diabetes descompensada sugirieron la posibilidad de diagnóstico, con confirmación final a través de la Prueba de supresión de dexametasona. La terapia se estableció con el uso de Trilostane, que demostró ser efectivo para controlar la patología. El objetivo de este informe de caso es mostrar que el hiperadrenocorticismo también afecta a los gatos, siendo una de las causas de diabetes en la especie.

Palabras clave: Felino, hiperadrenocorticismo, trilostane

\section{Introdução}

O Hiperadrenocorticismo (HAC) é uma endocrinopatia rara na rotina dos felinos, que acomete animais com a média de idade de 10 anos (Martinez \& Roca, 2009). De acordo com Mooney \& Shiel (2015), essa patologia se caracteriza por uma série de alterações clínicas e laboratoriais, resultante da produção excessiva de cortisol pelas glândulas adrenais, sendo a forma mais prevalente em gatos, ou resultado de uma administração crônica de glicocorticoides.

O hormônio liberador da corticotropina ( $\mathrm{CRH}$ ) estimula a adeno-hipófise a sintetizar e liberar o adrenocorticotrópico (ACTH), e este estimula a síntese e liberação de cortisol, que atua reduzindo a produção de ACHT e com isso auxilia no auto controle da concentração plasmática (Mooney \& Shiel, 2015). Em concentrações elevadas, tem efeito direto sobre o hipotálamo, diminuindo a secreção de CRH e sobre a adeno-hipófise, inibindo a secreção do ACTH (Feldman et al., 2014).

As manifestações clínicas frequentes são: poliúria, polidipsia, polifagia, perda de massa muscular, fragilidade cutânea, alopecia e infecções dermatológicas recorrentes (Mooney \& Shiel, 2015). Na maioria dos casos, a manifestação desses sinais clínicos ocorre juntamente com o aparecimento da Diabete Mellitus, fazendo com que o diagnóstico não seja precoce, podendo interferir no prognóstico (Nelson \& Couto, 2015).

De acordo com Martinez \& Roca (2009) e Nelson \& Couto (2015), o diagnóstico é através da anamnese, alterações físicas, avaliação dos exames laboratoriais e uso da ultrassonografia abdominal. Diferentemente dos cães, o exame complementar utilizado é o teste de supressão com dexametasona, o teste de estimulação com ACTH é mais utilizado para acompanhamento do tratamento. Seu tratamento pode ser clínico, com o uso do trilostano sendo prevalente, ou cirúrgico.

O objetivo desse trabalho foi relatar o caso clínico de um felino, pelo curto brasileiro, de 16 anos, com HAC, visto que esse diagnóstico é raro na rotina felina.

\section{Relato de caso}

No dia 29 de agosto do ano de 2019 foi atendido no Hospital Veterinário Intergávea, localizado no Jardim Botânico, Rio de Janeiro - RJ, um felino (Figura 1), macho, pelo curto brasileiro - PCB, pesando $2,9 \mathrm{~kg}$, com 16 anos apresentando um quadro de prostração associada a vômito e inapetência há 24 horas. No decorrer da consulta o tutor relatou que o paciente havia sido previamente diagnosticado diabético por outro médico veterinário, sem tratamento insulínico adequado. Durante o exame físico foi constatada a presença de intenso desconforto abdominal e aumento do abdômen, a pele estava delgada, o paciente apresentava desidratação acentuada, mucosas hipocoradas, temperatura $37,8{ }^{\circ} \mathrm{C}$, glicemia $224 \mathrm{mg} / \mathrm{dL}$ e pressão arterial $130 \mathrm{mmHg}$, também foi constatado a cetoacidose, através da gasometria e alterações clinicas compatíveis, e por esse motivo foi indicada a internação do paciente.

Foi realizado ultrassonografia (Figura 2), na qual foi observado a presença de uma imagem sugerindo um nódulo pancreático e pancreatite intensa, o intestino apresentava paredes finas com a camada muscular discretamente mais evidente, a bexiga apresentava paredes preservadas com a presença de um conteúdo anecogênico com sedimento hipoecogênico depositado na parede dorsal sugerindo cistite. Os rins apresentavam contornos irregulares e perda da definição córtico-medular, sugerindo uma nefropatia 
crônica bilateral associada a uma pielonefrite direita e as adrenais, medindo $1,27 \mathrm{~cm} \times 0,76 \mathrm{~cm}$ a direita e 1,46 cm x 0,64 cm a esquerda, com parênquima hipoecogênico e contornos abaulados sugerindo uma adrenomegalia bilateral. Na internação, o protocolo terapêutico utilizado foi fluidoterapia, protocolo de insulina glargina (IM e SC), Tramadol (2 mg/kg, BID), amoxicilina (20 mg/kg, BID), metronidazol (10 $\mathrm{mg} / \mathrm{kg}$, BID), dipirona $(25 \mathrm{mg} / \mathrm{kg}$ ) e ondansetrona $(1 \mathrm{mg} / \mathrm{kg})$.

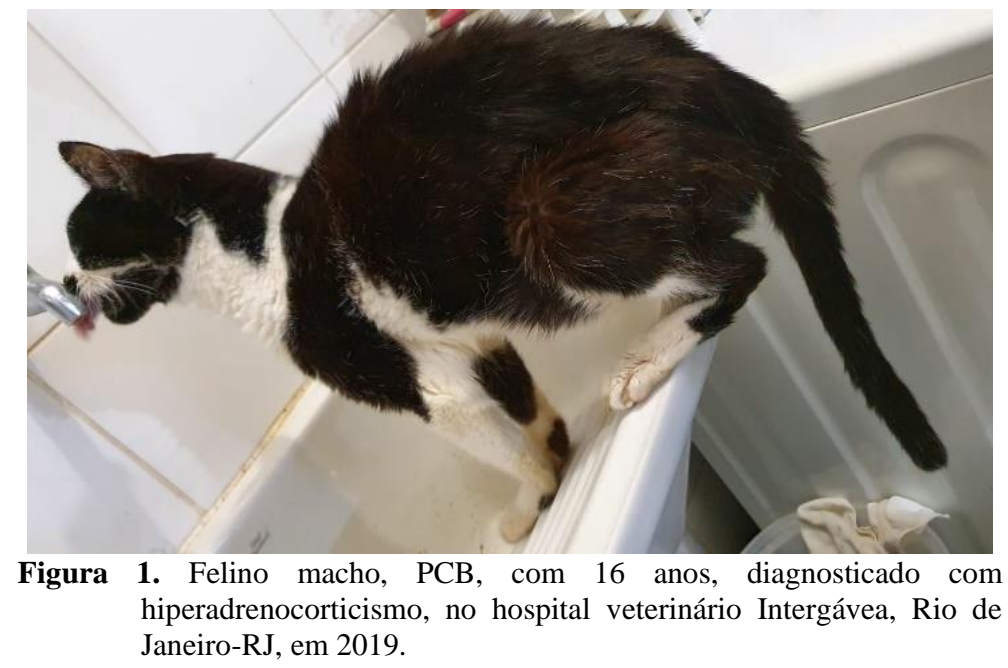

No segundo dia de internação, foram feitos os parâmetros e exame físico, constatando-se que a dor, desconforto abdominal e as mucosas hipocoradas persistiam, a temperatura estava $38^{\circ} \mathrm{C}$, glicemia média de $200 \mathrm{mg} / \mathrm{dL}$ e pressão arterial $185 \mathrm{mmHg}$.

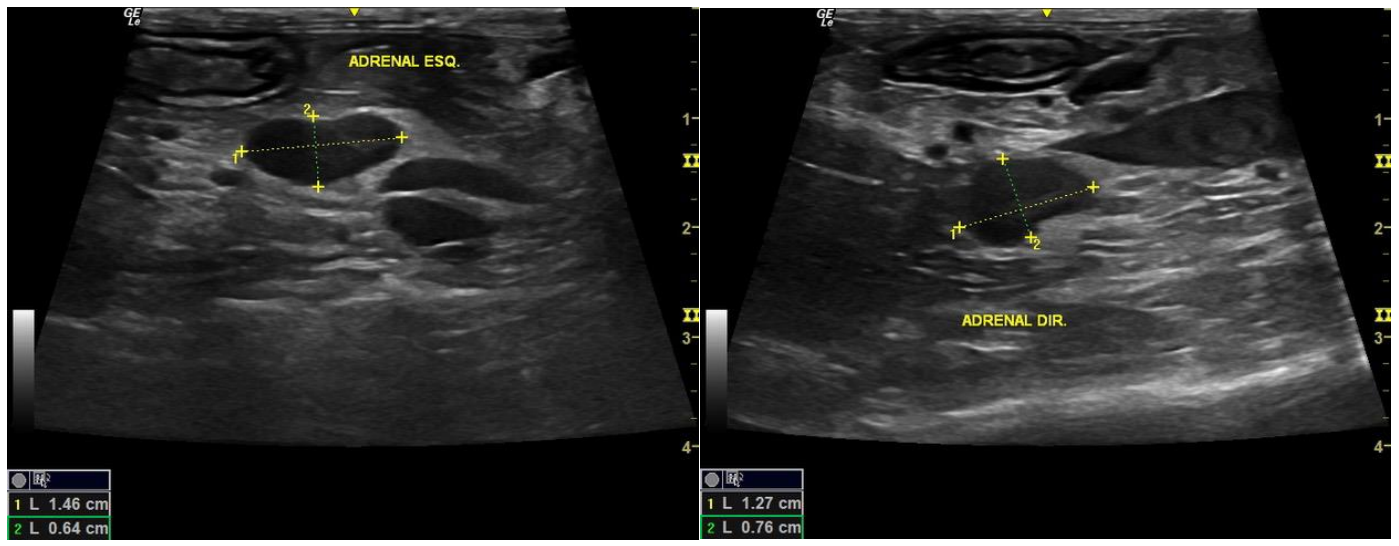

Figura 2. Imagens ultrassonográficas abdominais apresentando adrenais com parênquima hipoecogênico e contornos abaulados.

No terceiro dia, o paciente passou o plantão prostrado, porém responsivo a manipulação, se alimentou na presença da tutora (ração seca gastrointestinal) e após a visita ingeriu ração Diabetic. Houve maior consumo de água e consequentemente uma maior produção de urina. Durante a verificação dos parâmetros, observou-se que o animal apresentava um quadro de hipotermia, onde foram realizadas as manobras necessárias, havendo uma melhora. Foi também observado um quadro de hipertensão, logo foi administrado a medicação besilato de anlodipino $(0,625 \mathrm{mg})$ e após isso a pressão arterial se manteve entre 130 a $140 \mathrm{mmHg}$, o protocolo com glargina foi mantido. A análise hematológica revelou neutrofilia, linfopenia, monocitopenia absoluta e trombocitose (Tabela 1), além de um aumento nos valores de ureia, creatinina, ALT, potássio e albumina com valor abaixo do normal (Tabela 2).

Após quatro dias de internação, o paciente se encontrava prostrado, porém alerta, foi pouco responsivo durante o horário de visitas e ao decorrer do dia notou-se o aumento na ingestão de água e alimentos. Foi coletado material para urinálise através de cistocentese não guiada. No exame físico da urina foi observado uma coloração amarelo-clara, densidade levemente abaixo (1.012, sendo o valor de referência 1.020 - 1.060), e aspecto turvo. No exame químico, detectou-se glicosúria, presença de sangue 
oculto e pH levemente diminuído $(5,5$, sendo o valor de referência 6,0 - 7,0) e, na análise do sedimento foi detectado presença de hemácias e leucócitos. Os demais parâmetros foram aferidos e estavam dentro das normalidades, o material foi enviado para urocultura. Foi realizada a curva glicêmica, o paciente foi alimentado com ração recovery e diabetic.

Tabela 1. Resultados de hemogramas de um felino diagnosticado com hiperadrenocorticismo, nos dias 3 e 6 de tratamento, atendido no hospital veterinário Intergávea, Rio de Janeiro-RJ, em 2019.

\begin{tabular}{lccc}
\hline Eritrograma & Dia 3 & Dia 6 & Referência \\
\hline Eritrócitos $(\mathrm{x} 106 / \mu \mathrm{L})$ & 5,94 & 5,95 & 5,0 a 10,0 \\
Hemoglobina $(\mathrm{g} / \mathrm{dL})$ & 9,1 & 9,2 & 8,0 a 16,0 \\
Hematócrito $(\%)$ & 29,8 & 29,7 & 24,0 a 45,0 \\
VCM (fL) & 50,2 & 49,9 & 39,0 a 55,0 \\
CHGM $(\%)$ & 30,6 & 30,9 & 30,0 a 36,0 \\
Eritrócitos Nucleados (/100 Leucócitos) & 0 & 0 & 0 a 1 \\
Proteínas Plasmáticas Totais $(\mathrm{g} / \mathrm{dL})$ & 7,6 & $9,0+$ & 6,0 a 8,8 \\
\hline Leucograma & $(\mathrm{céls} / \mu \mathrm{L})$ & $($ céls/ $\mu \mathrm{L})$ & Referência \\
\hline Leucócitos & 17.900 & 25.200 & 5.500 a 19.500 \\
Basófilos & 0 & 252 & 0 a 300 \\
Eosinófilos & 1.074 & 252 & 100 a 1.500 \\
Mielócitos & 0 & 0 & 0 a 0 \\
Metamielócitos & 0 & 0 & 0 a 0 \\
Neutrófilos Bastonetes & 0 & 0 & 0 a 0 \\
Segmentados & $15.931+$ & $22.428+$ & 2.500 a 12.500 \\
Linfócitos & $895-$ & 2.016 & 1.500 a 7.000 \\
Monócitos & $0-$ & 252 & 100 a 850 \\
Plaquetas & $602.000+$ & $861.000+$ & 200.000 a 6000.000 \\
\hline
\end{tabular}

No quinto dia de tratamento, o animal continuou prostrado, porém responsivo a estímulos externos. A temperatura corporal estava $37,8^{\circ} \mathrm{C}$, a pressão arterial $130 \mathrm{~mm} / \mathrm{Hg}$ e a frequência cardíaca maior que $200 \mathrm{bpm}$. O paciente foi mantido no protocolo de insulina lantus. Foi recomendado avaliação com a endocrinologista, que percebeu alterações físicas de pele delgada, telangiectasia e aumento do volume abdominal, associado a informação ultrassonográfica de adrenomegalia. Foi solicitado então avaliação hormonal (teste de supressão por baixa de dexametasona), antibioticoterapia com enrofloxacina e ajuste da dose de insulina.

Tabela 2. Resultados das análises bioquímicas de um felino diagnosticado com hiperadrenocorticismo, nos dias 3 e 6 de tratamento, atendido no hospital veterinário Intergávea, Rio de Janeiro-RJ, em 2019.

\begin{tabular}{lccc}
\hline Exame & Dia 3 & Dia 6 & Referência \\
\hline ALT (U/L) & $151,9+$ & - & $6,0-83,0$ \\
Fosfatase Alcalina (U/L) & 14,8 & - & $10,0-90,0$ \\
Ureia (mg/dL) & $120,8+$ & $147,9+$ & $15,0-74,0$ \\
Creatinina Sérica (mg/dL) & $1,9+$ & $2,0+$ & $0,5-1,8$ \\
Proteína Total (g/dL) & 6,46 & - & $5,40-8,00$ \\
Albumina (g/dL) & $2,08-$ & - & $2,10-5,00$ \\
Globulina (g/dL) & 4,4 & - & $2,5-5,1$ \\
Relação Albumina Globulina & 0,47 & - & $0,4-1,3$ \\
Sódio (mmol/dL) & 155,0 & $146,0-$ & $147,0-156,0$ \\
Potássio (mmol/dL) & $5,4+$ & 4,3 & $4,0-4,5$ \\
Sódio Potássio & 28,70 & 33,95 & $27,3-38,1$ \\
Fósforo (mg/dL) & - & 4,3 & $4,2-8,1$ \\
Proteína Urinária (mg/dL) & - & 50,0 & - \\
Creatinina Urinária (mg/dL) & - & 27,5 & - \\
Relação PU/CU & - & 1,820 & - \\
\hline
\end{tabular}

Com seis dias de internação, o paciente estava menos prostrado, se alimentando e durante exame físico foi observado protrusão de terceira pálpebra em ambos os olhos, sem dor abdominal, ausculta sem 
alterações e mucosas normocoradas. A pressão arterial estava variando de 120 a $130 \mathrm{~mm} / \mathrm{Hg}$, estava hiperglicêmico $(258 \mathrm{mg} / \mathrm{dL})$ e os demais parâmetros se encontravam normais. A dose de lantus precisou ser novamente reajustada pata manter a glicemia de $100 \mathrm{a} 200 \mathrm{mg} / \mathrm{dL}$. Foi feito a coleta de amostra de sangue para realização de hemograma (Tabela 1) e análise bioquímica (Tabela 2). No sumário de urina, quando comparado ao primeiro exame, foi observado uma melhora da cor, a densidade permaneceu levemente abaixo (1.012, sendo o valor de referência 1.020 - 1.060), e o aspecto discretamente turvo. No exame químico, a glicose se apresentava normal, sangue oculto ainda presente, $\mathrm{pH}$ normalizado e, na análise do sedimento foi detectado ainda a presença de hemácias e leucócitos, mas em menores quantidades, e presença de bactérias.

No sétimo dia de internação, foram coletadas novas amostras de sangue para a realização dos exames hormonais, confirmando o diagnóstico (Tabela 3). O paciente foi liberado para casa com a seguinte terapêutica: Aplicação de $200 \mathrm{~mL}$ de soro ringer com lactato, via subcutâneo, na região dorsal próximo ao pescoço, a cada 24 horas durante 7 dias e após 7 dias a cada 48 horas. Como forma de controle, foi solicitado a realização da dosagem de glicemia pela manhã e noite, deixando avisado a necessidade de aplicação da insulina glargina (0,5 UI) caso apresente um valor igual ou superior a $150 \mathrm{mg} / \mathrm{dL}$, e após duas horas de aplicação, aferir novamente esse parâmetro. Foi estabelecido o protocolo com Vetoryl (1 $\mathrm{mg} / \mathrm{kg}$ ), trilostano manipulado, para controle do Hiperadrenocorticismo, realizada também a aplicação de cefovecina. Além disso, para a alimentação do paciente foi prescrito quatro sachês ultralight por dia. Foi solicitado a volta do paciente em cinco dias para outra avaliação.

Tabela 3. Resultados dos exames hormonais de um felino com hiperadrenocorticismo, no dia 7 de tratamento, atendido no hospital veterinário Intergávea, Rio de Janeiro-RJ, no ano de 2019.

\begin{tabular}{lccc}
\hline Data & Exame & Resultado & Valores normais \\
\hline $04 / 09 / 2019$ & Cortisol (Quimioluminescência) & $25,0 \mathrm{ng} / \mathrm{mL}$ & $\begin{array}{c}\text { Basal: } 10-30 \mathrm{ng} / \mathrm{mL} . \\
\text { (metodologia: Quimioluminescência) } \\
\text { 04/09/2019 }\end{array}$ \\
$\begin{array}{c}\text { Cortisol 8 horas pós dexametasona } \\
\text { (quimioluminescência) }\end{array}$ & $21,0 \mathrm{ng} / \mathrm{mL}$ & Pós dexametasona: $<10 \mathrm{ng} / \mathrm{mL}$ \\
\hline
\end{tabular}

Com três semanas de tratamento com trilostano ( $1 \mathrm{mg} / \mathrm{kg}$, BID) foi feito um novo exame dosando o cortisol pós administração de ACTH (Tabela 4), o paciente se encontra compensado da Diabetes, com média de glicemia de $100-150 \mathrm{mg} / \mathrm{dL}$, atualmente com 3,300 $\mathrm{kg}$ e com protocolo de insulina Lantus (1 UI, BID) e fluidoterapia para controle de doença renal.

Tabela 4. Resultado do teste de estimulação pós ACTH de um felino com hiperadrenocorticismo, com três semanas de tratamento com trilostano (1 mg/kg, BID), atendido no hospital veterinário Intergávea, Rio de Janeiro-RJ, no ano de 2019

\begin{tabular}{|c|c|c|c|}
\hline Data & Exame & Resultado & Valores normais \\
\hline $01 / 10 / 2019$ & Cortisol (Quimioluminescência) & $19,0 \mathrm{ng} / \mathrm{mL}$ & $\begin{array}{l}\text { Basal: } 10 \text { - } 30 \mathrm{ng} / \mathrm{mL} \text {. } \\
\text { (metodologia: Quimioluminescência) }\end{array}$ \\
\hline $01 / 10 / 2019$ & $\begin{array}{l}\text { Cortisol pós ACTH } \\
\text { (quimioluminescência) }\end{array}$ & $33,0 \mathrm{ng} / \mathrm{mL}$ & $\begin{array}{c}\text { Normal: } 50 \text { - } 100 \mathrm{ng} / \mathrm{mL} \text { (metodologia: } \\
\text { Quimioluminescência) }\end{array}$ \\
\hline
\end{tabular}

\section{Discussão}

O hiperadrenocorticismo surge geralmente em gatos de meia idade a idosos, corroborando com os achados do presente caso, onde o paciente tem 16 anos. Ainda de acordo com Feldman et al. (2014), não existe predisposição racial ou sexual. Em felinos essa patologia é rara, tendo poucos relatos publicados.

O paciente já havia sido diagnosticado com Diabetes Mellitus (DM), o que na maioria dos casos ocorre porque os tutores ou veterinários só observam após desenvolvimento dessa patologia, por isso normalmente o diagnóstico é tardio e quando está em uma fase avançada. Segundo Feldman et al. (2014), a maioria dos gatos com essa patologia apresentam concomitante com DM. Normalmente, os sintomas que encaminham os tutores com seus animais a clínica são poliúria, polidipsia e polifagia. No caso de gatos previamente diagnosticado com DM, estes três sinais são associados pelos proprietários a dificuldade de regulação desta patologia. 
No exame físico as principais alterações que chamam atenção são abdômen pendular, atrofia muscular generalizada e manifestações dermatológicas, como alopecia, atrofia cutânea, lesões cutâneas, seborreia e hiperpigmentação. No caso do felino relatado, o que chamou atenção foi a pele fina e aumento da região abdominal, o que levou a suspeita de HAC. Segundo Feldman et al. (2014), ao nível do tecido adiposo, o cortisol em excesso promove a estimulação de enzimas lipolíticas, conduzindo ao aumento da taxa de lipólise e a redistribuição da gordura para o abdômen e para o fígado, isso provoca pressão sobre a musculatura enfraquecida e a aparência de abdômen pendular (Cunningham, 2014).

Existem ainda alterações dermatológicas que são efeitos dos glucocorticoides, que exercem na pele efeitos atróficos na epiderme e derme, levando a maior propensão para lesões da pele e a má cicatrização dessas lesões (Ramos, 2011). Uma alteração específica do gato é a síndrome da pele frágil, que causa perda de elasticidade e que rasga com facilidade. Essas alterações dermatológicas podem resultar do excesso de glucocorticoides endógenos como exógenos e sua presença contribuiu negativamente para prognóstico dos felinos.

Avaliação laboratorial no gato tem resultados inconsistentes, o que dificulta o reconhecimento da síndrome de Cushing. Os resultados podem não revelar alterações específicas que sejam úteis no diagnóstico (Duesberg \& Peterson, 1997), contudo essa avaliação auxilia na exclusão de outras causas. Na hematologia podem ser observados alterações como neutrofilia e monocitose, devido à diminuição da marginalização destas células e diminuição da diapedese dos neutrófilos nos tecidos e eosinopenia devido ao sequestro destas células na medula óssea. Isso se deve a um dos efeitos dos glucocorticoides que atua na movimentação das células do sistema imunitário. O leucograma de estresse apresenta neutrofilia, monocitose, linfopenia e eosinopenia em gatos, não tem sido consistente, sendo mais presente em cães (Furuzawa et al., 1992; Peterson \& Steele, 1986).

Nos parâmetros bioquímicos, pode ser observado que não há uma variação significativa dos valores de ureia e creatinina, embora Feldman et al. (2014) tenham descrito valores abaixo dos níveis normais, porém o gato relatado tinha doença renal, por isso tais alterações.

Nas enzimas hepáticas, de acordo com Duesberg \& Peterson (1997) um pequeno aumento da atividade da alanino-aminotransferase (ALT) foi encontrado em $40 \%$ dos gatos com hiperadrenocorticismo e $20 \%$ apresentam aumento da FAS, essa porcentagem se deve pela meia-vida da FAS em gatos que é de apenas 7 horas, e eles não possuem a isoenzima induzida por glucocorticoides (Feldman et al., 2014), como no presente caso.

Na urinálise, os gatos frequentemente apresentam valores elevados, apesar da poliúria e polidipsia presentes (Ettinger \& Feldman, 2004). Não existem evidências que os gatos apresentem síndrome semelhante à dos cães, assim foi sugerido que a poliúria e a polidipsia resultam da DM concomitante ou, em alguns casos, de insuficiência renal crônica (Feldman et al., 2014), como no paciente relatado. Os felinos ainda podem apresentar evidências de infecção do trato urinário e podem também apresentar proteinúria, por perda sobretudo de albumina, secundária a alterações glomerulite (Feldman et al., 2014; Peterson \& Steele, 1986).

Nas avaliações de imagens, pôde ser feito o uso de várias técnicas, nas alterações radiológicas é possível visualizar bom contraste radiográfico resultante da deposição de gordura no mesentério, o abdômen pendular, redução da musculatura lombar e a distensão da bexiga (Feldman et al., 2014). A ecografia tem auxiliado no diagnóstico, permitindo localização das glândulas com facilidade. A ultrassonografia das glândulas adrenais pode ser utilizada para avaliar o tamanho e forma, já que nos felinos é comum encontrar adenomegalia adrenal bilateral como consequência do excesso de ACTH e felinos com ambas as glândulas adrenais aumentadas ou de igual tamanho, sendo o diagnóstico é HAC hipófise dependente (Martinez \& Roca, 2009; Mooney \& Shiel, 2015), sendo essa opção a de escolha para o paciente relatado. A largura máxima da glândula adrenal nos felinos saudáveis é tipicamente menor que $0,5 \mathrm{~cm}$ e deve suspeitar de adrenomegalia quando a largura máxima for maior que $0,5 \mathrm{~cm}$, altura máxima maior que $0,8 \mathrm{~cm}$, o que nos é indicado no laudo do paciente, já que ambas estão aumentadas e hipoecogênicas. Ainda poder ser feito o uso de tomografia computadorizada e ressonância magnética, que permitem visualização de alterações nas glândulas e tumores hipofisários.

O diagnóstico nos felinos é complexo, porém existem três teste laboratoriais utilizados: teste de estimulação com $\mathrm{ACTH}$, teste de supressão pela dexametasona em baixa dose e proporção cortisol: 
creatinina urinária. O teste de supressão pela dexametasona em dose baixa, ele avalia a capacidade de supressão do eixo HHA (Feldman et al., 2014). A dose administrada em gatos para realização do teste é mais elevada do que a usada em cães $(0,01 \mathrm{mg} / \mathrm{kg})$, isso ocorre porque foi verificado que a dose usada em cães era menos sensíveis em gatos (Ettinger \& Feldman, 2004), assim foi indicado a utilização de $0,1 \mathrm{mg} / \mathrm{kg}$ de dexametasona como teste diagnóstico em gatos. Esse teste é altamente sensível, então mais indicado para identificar hiperadrenocorticismo em felinos (Feldman et al., 2014; Mooney, 1998). Neste relato os dois primeiros testes foram utilizados, sendo o teste de supressão usado primeiro para diagnóstico, dando positivo e logo após o teste de estimulação para continuação do tratamento.

O tratamento para hiperadrenocorticismo varia entre tratamento médico e cirúrgico. Sendo o cirúrgico a adrenalectomia unilateral ou bilateral, o que não foi indicado no relato já que o paciente apresentava hiperplasia bilateral das adrenais. Os tratamentos medicamentosos são utilizados de acordo com os seus locais de ação farmacológica. Alguns atuam nas glândulas adrenais inibindo a esteroidogênese, há os que atuam diretamente no tumor e por fim aqueles cuja função é de bloquear os receptores dos glicocorticoides (Constanzo \& Picó, 2012). Temos como opções o Mitotano, o Etomidato e o Trilostano, sendo ele a melhor opção terapêutica para HAC em felinos (Martinez \& Roca, 2009; Scudder et al., 2015). O trilostano foi o tratamento de escolha para o presente relato, já que ele inibe a enzima 3- $\beta$-hidroxiesteroide-desidrogenase (3 $\beta$-HAD), responsável pela conversão da pregnenolona em progesterona, inibindo a conversão da progesterona em cortisol e aldosterona (Neiger et al., 2004; Skelly et al., 2003).

\section{Conclusão}

O Hiperadrenocorticismo é uma doença endócrina rara na rotina dos felinos, considerada grave, de prognóstico reservado a desfavorável, uma vez que o diagnóstico é tardio e observado concomitante a Diabetes Mellitus, em fase avançada. $\mathrm{O}$ teste de supressão por baixa dose de dexametasona é o método de diagnóstico de eleição em felinos suspeitos com síndrome de Cushing. O tratamento com o uso de trilostano demonstrou ser uma boa opção, uma vez que houve uma melhora na condição geral do paciente, sendo o tratamento clínico de escolha, até o presente momento, de tratamento para o Hiperadrenocorticismo felino.

\section{Referências}

Constanzo, M.-J. B., \& Picó, C. del P. (2012). Nuevas perspectivas en el tratamiento farmacológico de la enfermedad de Cushing. Endocrinología y Nutrición, 59(10), 599-605. https://doi.org/10.1016/j.endonu.2012.07.008

Cunningham, J. (2014). Tratado de fisiologia veterinária (Elsevier). Guanabara Koogan.

Duesberg, C., \& Peterson, M. E. (1997). Adrenal Disorders in Cats. Veterinary Clinics of North America: Small Animal Practice, 27(2), 321-347. https://doi.org/10.1016/S0195-5616(97)50035-0

Ettinger, S. J., \& Feldman, E. C. (2004). Tratado de medicina interna veterinária: doenças do cão e do gato. In (5o Edição). Rio de Janeiro: Editora Guanabara Koogan S.A.

Feldman, E. C., Nelson, R. W., Reusch, C., \& Scott-Moncrieff, J. C. (2014). Canine and feline endocrinology. Elsevier Health Sciences.

Furuzawa, Y., Une, Y., \& Nomura, Y. (1992). Pituitary Dependent Hyperadrenocorticism in a Cat. The Journal of Veterinary Medical Science, 54(6), 1201-1203. https://doi.org/10.1292/jvms.54.1201

Martinez, M., \& Roca, A. L. (2009). Hiperadrenocorticismo felino tratado con trilostano. Clínica Veterinaria de Pequeños Animales, 29(4), 255.

Mooney, C. (1998). Unusual endocrine disorders in the cat. In Practice, 20(7), 345-349. https://doi.org/10.1136/inpract.20.7.345

Mooney, C. T., \& Shiel, R. E. (2015). Hipotireoidismo em cães. Manual de Endocrinologia Em Cães e Gatos, 81-82.

Neiger, R., Witt, A. L., Noble, A., \& German, A. J. (2004). Trilostane Therapy for Treatment of Pituitary-Dependent Hyperadrenocorticism in 5 Cats. Journal of Veterinary Internal Medicine, 18(2), 160-164. https://doi.org/10.1111/j.1939-1676.2004.tb00156.x

Nelson, R., \& Couto, C. G. (2015). Medicina interna de pequenos animais. Elsevier Brasil. 
Peterson, M. E., \& Steele, P. (1986). Pituitary-dependent hyperadrenocorticism in a cat. Journal of the American Veterinary Medical Association, 189(6), 680-683.

Ramos, M. L. (2011). Hiperadrenocorticismo felino: revisão bibliográfica e revisão de caso [Universidade Técnica de Lisboa. Faculdade de Medicina Veterinária]. http://hdl.handle.net/10400.5/2946

Scudder, C., Kenny, P., \& Niessen, S. (2015). Treatment of canine and feline hyperadrenocorticism: trilostane and the alternatives. Companion Animal, 20(4), 230-238. https://doi.org/10.12968/coan.2015.20.4.230

Skelly, B. J., Petrus, D., \& Nicholls, P. K. (2003). Use of trilostane for the treatment of pituitarydependent hyperadrenocorticism in a cat. Journal of Small Animal Practice, 44(6), 269-272. https://doi.org/10.1111/j.1748-5827.2003.tb00154.x

\section{Histórico do artigo:}

Recebido: 1 de setembro de 2020 .

Aprovado: 5 de outubro de 2020.

Disponível online: 18 de novembro de 2020.
Licenciamento: Este artigo é publicado na modalidade Acesso Aberto sob a licença Creative Commons Atribuição 4.0 (CC-BY 4.0), a qual permite uso irrestrito, distribuição, reprodução em qualquer meio, desde que o autor e a fonte sejam devidamente creditados. 